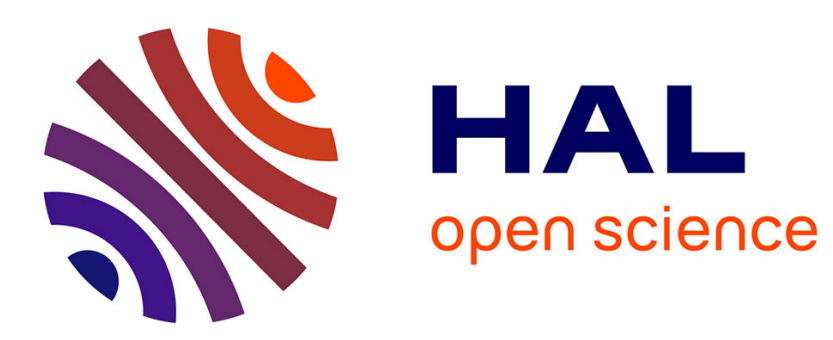

\title{
On Lower Bounds of the Density of Delone Sets and Holes in Sequences of Sphere Packings
}

\author{
Gilbert Muraz, Jean-Louis Verger-Gaugry
}

\section{To cite this version:}

Gilbert Muraz, Jean-Louis Verger-Gaugry. On Lower Bounds of the Density of Delone Sets and Holes in Sequences of Sphere Packings. Experimental Mathematics, 2005, 14 (1), pp.47-57. hal-00346420

\author{
HAL Id: hal-00346420 \\ https://hal.science/hal-00346420
}

Submitted on 9 Jan 2009

HAL is a multi-disciplinary open access archive for the deposit and dissemination of scientific research documents, whether they are published or not. The documents may come from teaching and research institutions in France or abroad, or from public or private research centers.
L'archive ouverte pluridisciplinaire HAL, est destinée au dépôt et à la diffusion de documents scientifiques de niveau recherche, publiés ou non, émanant des établissements d'enseignement et de recherche français ou étrangers, des laboratoires publics ou privés. 


\title{
On Lower Bounds of the Density of Delone Sets and Holes in Sequences of Sphere Packings
}

\author{
G. Muraz and J.-L. Verger-Gaugry
}

September 21, 2004

\begin{abstract}
We study lower bounds of the packing density of a system of nonoverlapping equal spheres in $\mathbb{R}^{n}, n \geq 2$, as a function of the maximal circumradius of its Voronoi cells. Our viewpoint is that of Delone sets which allows to investigate the gap between the upper bounds of Rogers or Kabatjanskii-Levenštein and the Minkowski-Hlawka type lower bounds for the density of lattice-packings, without entering the fundamental problem of constructing Delone sets with Delone constants between $2^{-0.401}$ and 1 . As a consequence we provide explicit asymptotic lower bounds of the covering radii (holes) of the BarnesWall, Craig and Mordell-Weil lattices, respectively $B W_{n}, \mathbb{A}_{n}^{(r)}$ and $M W_{n}$, and of the Delone constants of the BCH packings, when $n$ goes to infinity.
\end{abstract}

2000 Mathematics Subject Classification: 52C17, 52C23.

Keywords : Delone set, sphere packing, density, hole.

\section{INTRODUCTION}

The maximal packing density of equal spheres in $\mathbb{R}^{n}$ has received a lot of attention [Rogers 1964], [Handbook 1997], [Cassels 1959], [Martinet 1996], [Conway - Sloane 1988], [Oesterlé 1989/90], [Gruber - Lekkerkerker 1987], [Zong 1999]. Similar problems are encountered in coding theory, data transmission, combinatorial geometry and cryptology [Hoffstein 2001]. We will consider it by the notion of Delone set for which we will give explicit lower bounds of the density of a Delone set as a function of $n$ and its so-called Delone constant $R$ expressing the maximal size of its holes.

Blichfeldt, Rogers, Levenštein, Sidel'nikov, Kabatjanskii and Levenštein [Handbook 1997] [Gruber - Lekkerkerker 1987] [Conway - Sloane 1988] have given upper bounds while lower bounds were given by Minkowski, DavenportRogers, Ball [Ball 1992], etc, in the lattice-packing case (see Section 2). In between the situation is considered as fairly vague. The present note is a contribution to the range between both types of bounds though the fundamental problem, far from obvious, of constructing Delone sets of very small Delone constant, namely less than 1 is not considered here. 
For this we will recall the language of uniformly discrete sets and Delone sets instead of that of systems of spheres. A discrete subset $\Lambda$ of $\mathbb{R}^{n}$ is said to be uniformly discrete if there exists a constant $r>0$ such that $x, y \in \Lambda, x \neq y$ implies $\|x-y\| \geq r$. Thus a uniformly discrete set is either the empty set, a subset $\{x\}$ reduced to one element or, if it contains at least two points, they satisfy such an inequality; if $r$ is equal to the minimal interpoint distance $\inf \{\|x-y\| \mid x, y \in \Lambda, x \neq y\}, \Lambda$ is said to a uniformly discrete set of constant $r$ of $\mathbb{R}^{n}$. Uniformly discrete sets of constant 1 will be called $\mathcal{U D}$-sets and the set of $\mathcal{U D}$-sets will be denoted by $\mathcal{U D}$ (without mentioning the dimension $n$ of the ambiant space). There is a one-to-one correspondence between the set $S S$ of systems of equal spheres of radius $1 / 2$ and the set $\mathcal{U D}: \Lambda=\left(a_{i}\right)_{i \in \mathbb{N}} \in \mathcal{U D}$ is the set of sphere centres of $\mathcal{B}(\Lambda)=\left\{a_{i}+B \mid i \in \mathbb{N}\right\} \in S S$ where $B(z, t)$ denotes generically the closed ball centred at $z \in \mathbb{R}^{n}$ of radius $t>0$, and $B:=B(0,1 / 2)$. We will take $1 / 2$ in the sequel for the common radius of spheres to be packed and will consider $\mathcal{U D}$-sets instead of systems of equal spheres of radius $1 / 2$.

Let $\Lambda \in \mathcal{U D}$. The density of the system of spheres $\mathcal{B}(\Lambda)$ is defined by

$$
\delta(\mathcal{B}(\Lambda)):=\limsup _{R \rightarrow+\infty}\left[\operatorname{vol}\left(\left(\bigcup_{i \in \mathbb{N}}\left(a_{i}+B\right)\right) \bigcap B(0, R)\right) / \operatorname{vol}(B(0, R))\right]
$$

Let us denote by $\mathcal{L}$ the space of ( $n$-dimensional) lattices of $\mathbb{R}^{n}$. We will denote

$$
\delta:=\sup _{\Lambda \in \mathcal{U} \mathcal{D}} \delta(\mathcal{B}(\Lambda)), \quad \text { resp. } \quad \delta_{L}:=\sup _{\Lambda \in \mathcal{L} \cap \mathcal{U} \mathcal{D}} \delta(\mathcal{B}(\Lambda))
$$

and will call them respectively the packing density and the lattice-packing density.

A $\mathcal{U D}$-set $\Lambda$ is said to be a Delone set if there exists a constant $R>0$ such that, for all $z \in \mathbb{R}^{n}$, there exists an element $\lambda \in \Lambda$ such that $\|z-\lambda\| \leq R$ (property of relative denseness of Besicovitch). If $\Lambda$ is a Delone set, then $R(\Lambda):=\sup _{z \in \mathbb{R}^{n}} \inf _{\lambda \in \Lambda}\|z-\lambda\|$ is called the Delone constant of $\Lambda$. Let $R_{c}=R_{c}(n):=\inf \{R(\Lambda) \mid \Lambda \in \mathcal{U D}\}$. This lower bound is an invariant of the ambiant space which is only a function of $n$ and the Euclidean metric on $\mathbb{R}^{n}$. We will call it the Delone covering constant.

In Section 2 we will recall the asymptotic expressions of the classical upper bounds of the packing density and the lower bounds of the latticepacking density, when $n$ goes to infinity.

In Section 3 we will recall known lower bounds of the minimal hole constant, in the case of lattice packings, and state some results concerning lower bounds of $R_{c}$ in the general case of arbitrary packings. 
The Delone constant of a Delone set $\Lambda \in \mathcal{U D}$ is the maximal circumradius of the Voronoi cells in the Voronoi decomposition of space by $\Lambda$ (Section 3); if $\Lambda$ is a lattice, it is the covering radius of the lattice, if $\Lambda$ is a non periodic $\mathcal{U D}$-set, it is the "maximal size of the holes in $\Lambda$ ". In Section 4 , we will prove

Theorem 1.1 Let $n \geq 2$. If $\Lambda$ is a Delone set of $\mathbb{R}^{n}$ of Delone constant $R$, then

$$
(2 R)^{-n} \leq \delta(\mathcal{B}(\Lambda)) \leq \delta \quad \text { for all } \quad R_{c} \leq R
$$

Let us denote $\mu_{n}(R):=(2 R)^{-n}$. The $(2 R)^{-n}$ dependence of the expression of $\mu_{n}(R)$ with $n$ is very important and constitutes a key result. It allows to study the minimal asymptotic values of the Delone covering constant $R_{c}(n)$ when $n$ tends to infinity. Namely, we will prove

Theorem 1.2 For all $\epsilon>0$ there exists $n(\epsilon)$ such that for $n>$ $n(\epsilon), R_{c}(n) \geq 2^{-0.401}-\epsilon$.

REMARK.- Theorem 1.2 asserts the existence of an infinite collection of middle-sized Voronoi cells in any densest or saturated packing of equal spheres of $\mathbb{R}^{n}$ of radius $1 / 2$ of circumradii greater than

$$
2^{-0.401}+o(1)=0.757333 \ldots+o(1) .
$$

The small values of $R$ between the bound $\frac{\sqrt{2}}{2} \sqrt{\frac{n}{n+1}}$ and 1 are discussed in Section 3.

In Section 5, as an application of Theorem 1.1, we will obtain explicit lower bounds as a function of $n$ of the covering radii (holes) of known lattices, namely Barnes-Wall $B W_{n}$, Craig $\mathbb{A}_{n}^{(r)}$, Mordell-Weil $M W_{n}$, and of the Delone constants of $\mathrm{BCH}$ packings.

In Section 6 we will show the pertinency of the lower bound $\mu_{n}(R)$, and "its continuity with $R$ ", by comparing it to known classical asymptotic bounds. The construction of Delone sets of very small Delone constants is a difficult problem which is not considered here. Concerning lattice packings, our results give credit to the Conjecture stating that: for all $\epsilon>0$, there exists $n_{L}(\epsilon)$ such that for $n>n_{L}(\epsilon)$, for all ( $n$-dimensional) lattice $L \in \mathcal{U D}, R(L) \geq 1-\epsilon$. 


\section{ASYMPTOTIC BEHAVIOUR OF THE UPPER BOUNDS OF $\delta$ AND OF THE LOWER BOUNDS OF $\delta_{L}$}

The upper bounds of $\delta$, as a function of $n$, are recalled in Table 1 , the best one being the one of Kabatjanskii and Levenštein ([Rogers 1964], [Gruber - Lekkerkerker 1987], §19 and §38, p 390-391, [Conway - Sloane 1988], Chap. 1 and 9, [Zong 1999], Chap. 3).

Table 1

\begin{tabular}{l|l}
\hline$c n 2^{-n / 2}(c$ a const.) & Blichfeldt [Blichfeldt 1929] \\
$\frac{n}{e} 2^{-n / 2}$ & Rogers [Rogers 1958] \\
$2^{(-0.5096+o(1)) n}$ & Sidel'nikov [Sidel'nikov 1973] \\
$2^{(-0.5237+o(1)) n}$ & Levenštein [Levenštein 1979] \\
$2^{(-0.5990+o(1)) n}$ & Kabatjanskii and Levenštein [Kabatjanskï - Levenštein 1978]
\end{tabular}

Their asymptotic expressions, when $n$ goes to infinity, all exhibit a dominant exponential term of the type $2^{-\alpha n}$ where $\alpha$ is close to $1 / 2$. As for lower bounds, non-trivial lower bounds of the packing constant $\delta$ do not seem to exist yet (see Section 6; [ElkiesI 2000]). The basic result is concerned with lattice packings: the Conjecture of Minkowski (1905) proved by Hlawka [Cassels 1959] [Gruber - Lekkerkerker 1987] states

$$
\frac{\zeta(n)}{2^{n-1}} \leq \delta_{L}
$$

where $\zeta(n)=\sum_{k=1}^{\infty} k^{-n}$ denotes the Riemann $\zeta$-function. This lower bound is non-effective since its proof does not allow explicit constructions of very dense lattices. This lower bound was improved by Davenport and Rogers [Davenport - Rogers 1947] who gave: $(\ln \sqrt{2}+o(1)) n 2^{-n}$, for $n$ sufficiently large, and by Ball [Ball 1992] who recently obtained better: $2(n-1) \zeta(n) 2^{-n}$. For details, see [Handbook 1997], Chap. VI in Cassels [Cassels 1959], [Conway - Sloane 1988] Chap. 9, [Gruber - Lekkerkerker 1987], or [Zong 1999]. One can remark that these asymptotic expressions all exhibit a dominant exponential term in $2^{-\alpha^{\prime} n}$ with $\alpha^{\prime}=1$, and that there exists a close similarity between the asymptotic expressions of the lower and upper bounds and Theorem 1.1. Theorem 1.1 will allow to "go continuously" in some sense from the first type ( " $\alpha \simeq 1 / 2$ " case) to the second type (" $\alpha^{\prime}=1 "$ case) of bounds; see Section 6 . 


\section{LOWER BOUNDS OF THE MINIMAL HOLE CONSTANT $R_{L}(n)$ AND OF THE DELONE COVERING CONSTANT $R_{c}(n)$}

Bounds for the (lattice-)packing density are obviously linked to holes. Let us recall some definitions. If a lattice $\Lambda \in \mathcal{U D}$ of $\mathbb{R}^{n}$ is a Delone set of Delone constant $R$, then classically the quantity $R$ is called the covering radius of $\Lambda$. Given a $\mathcal{U D}$-set $\Lambda:=\left\{\lambda_{i}\right\}$, to each element $\lambda_{i} \in \Lambda$ is associated its local cell $C\left(\lambda_{i}, \Lambda\right)$, also denoted by $C\left(\lambda_{i}, \mathcal{B}(\Lambda)\right)$, defined by the closed subset (not necessarily bounded), called Voronoi cell at $\lambda_{i}$,

$$
C\left(\lambda_{i}, \Lambda\right):=\left\{x \in \mathbb{R}^{n} \mid\left\|x-\lambda_{i}\right\| \leq\left\|x-\lambda_{j}\right\| \text { for all } j \neq i\right\} .
$$

As soon as $\Lambda$ is a Delone set of Delone constant $R>0(R<+\infty)$, all the Voronoi cells at its points are bounded closed convex polyhedra. In this case, for all $\lambda_{i} \in \Lambda$, we have

$C\left(\lambda_{i}, \Lambda\right):=\left\{x \in \mathbb{R}^{n} \mid\left\|x-\lambda_{i}\right\| \leq\left\|x-\lambda_{j}\right\|\right.$ for all $j \neq i$ with $\left.\left\|\lambda_{j}-\lambda_{i}\right\|<2 R\right\}$.

By definition the circumradius of the Voronoi cell at $\lambda_{i}$ is $\rho_{i}:=\max _{v} \| \lambda_{i}-$ $v \|$ where the supremum (reached) is taken over all the vertices $v$ of the Voronoi cell $C\left(\lambda_{i}, \Lambda\right)$ at $\lambda_{i}$ and the Delone constant $R$ of $\Lambda$ is equal to $\max _{i} \rho_{i}$. The elements $z \in \mathbb{R}^{n}$ lying at a distance $R(\Lambda)$ of $\Lambda$ will be called (spherical) deep holes (or deepest holes) of $\Lambda$. The other vertices of Voronoi cells will be called holes.

In the particular case of a lattice $L$ the covering radius $R(L)$ is the circumradius of the Voronoi cell of the lattice $L$ at the origin. Any vertex of this Voronoi cell at a distance of $L$ less than $R(L)$ from $L$ is called shallow hole [Conway - Sloane 1988]. All the vertices of the Voronoi cell of a lattice at the origin may be simultaneously deepest holes when this Voronoi cell is highly symmetrical [Verger-Gaugry 1997].

Let us define the minimal hole constant by

$$
R_{L}=R_{L}(n):=\min _{L \in \mathcal{U} \mathcal{D} \cap \mathcal{L}} R(L)
$$

over all lattices $L$ of $\mathbb{R}^{n}$ which are $\mathcal{U D}$-sets. Its determination is an important problem, already mentioned by Fejes-Toth [Fejes-Toth 1979]. It corresponds to the smallest possible holes in lattice packings $L+B$. Our knowledge about it is comparatively limited and the lattices for which the covering radius is equal to the minimal hole constant are unknown as soon as $n$ is large enough. In Table 2 we summarize some values and known upper bounds of $R_{L}(n)$. 
Table 2 : Minimal hole constant $R_{L}(n)$ for lattice-packings of spheres of

\begin{tabular}{lcl}
\multicolumn{4}{c}{ radius 1/2 } & in $\mathbb{R}^{n}$. \\
\hline$n=3$ & Böröczky [Böröczky 1986] & $=\sqrt{5} /(2 \sqrt{3}) \simeq 0.645497 \ldots$ \\
$n=4$ & Horvath [Horvath 1982] & $=(\sqrt{3}-1) 3^{1 / 4} / \sqrt{2} \simeq 0.68125 \ldots$ \\
$n=5$ & Horvath [Horvath 1982] & $=\sqrt{9+\sqrt{13}} /(2 \sqrt{6}) \simeq 0.72473 \ldots$ \\
$n \geq 2$ & Rogers [Rogers 1950] & $<1.5$ \\
$n \geq 2$ & Henk [Henk 1995] & $\leq \sqrt{21} / 4 \simeq 1.1456 \ldots$ \\
$n>>1$ & Butler [Butler 1972] & $\leq n^{\left(\log _{2} \ln n+c\right) / n}=1+o(1)(c$ is a constant $)$
\end{tabular}

The following Theorem is fundamental but non-constructive.

Theorem 3.1 ( Butler )

$$
R_{L}(n) \leq 1+o(1) \quad \text { when } n \text { is sufficiently large. }
$$

This leads to the following question:

$\left(\mathrm{Q}_{1}\right)$ For all $\epsilon>0$, does there exist $n_{0}(\epsilon)$ such that the inequality $R_{L}(n) \geq 1-\epsilon$ holds for all $n \geq n_{0}(\epsilon)$ ?

If the answer to this fundamental question is yes, then Butler's Theorem [Butler 1972] would imply that $R_{L}(n)=1+o(1)$. Then this result would be a very important step towards a proof of the Conjecture stating that the strict inequality " $\delta>\delta_{L}$ " holds for $n$ large enough. The affirmative answer to question $\left(\mathrm{Q}_{1}\right)$ is a Conjecture [Conway - Sloane 1988]. Consequently, the search for lower bounds of $R_{L}(n)$ is crucial.

The lower bound $\sqrt{2} / 2+o(1)$ for $R_{L}(n)$ was given by Blichfeldt (Butler [Butler 1972] p 722), when $n$ is large enough. Let us note that the normalized (see Section 5) Leech lattice $\Lambda_{24} / \sqrt{N\left(\Lambda_{24}\right)}$ [ElkiesII 2000] has a small value of its covering radius by the Theorem of Conway, Parker and Sloane (in [Conway - Sloane 1988] Chap. 23): $R\left(\Lambda_{24} / \sqrt{N\left(\Lambda_{24}\right)}\right)=\sqrt{2} / 2$. In low dimension, this value is rarely reached [Conway - Sloane 1988]. In general, for lattices, the information about its holes is limited Chap. 22 by Norton in [Conway - Sloane 1988]) because of the difficulty of computing explicitely the Voronoi cells of a lattice from the lattice itself when $n$ is large.

Let us now turn to the notion of saturation, linked to the possible filling of holes. We will say that a $\mathcal{U D}$-set $\Lambda$ is saturated, or maximal, if it is impossible to add a sphere to $\mathcal{B}(\Lambda)$ without destroying the fact that it is a packing of spheres, i.e. without creating an overlap of spheres. The set $S S$ of systems of spheres of radius $1 / 2$, is partially ordered by the relation $\prec$ defined by

$$
\Lambda_{1}, \Lambda_{2} \in \mathcal{U D}, \quad \mathcal{B}\left(\Lambda_{1}\right) \prec \mathcal{B}\left(\Lambda_{2}\right) \Longleftrightarrow \Lambda_{1} \subset \Lambda_{2} .
$$


By Zorn's Lemma, maximal sphere packings exist. The saturation operation of a sphere packing consists in adding spheres to obtain a maximal sphere packing. It is fairly arbitrary and may be finite or infinite. Note that it is not because a sphere packing is maximal (saturated) that its density is equal to $\delta$.

Let $X_{R} \subset \mathcal{U D}$ be the subset of Delone sets of Delone constant $R>0$ of $\mathbb{R}^{n}$. By saturating a Delone set of Delone constant $R>0$ we will always obtain a Delone set of constant less than 1 , but not a Delone set of Delone constant $=R_{c}$ in general. Let $R^{(s)}:=\sup \{R(\Lambda) \mid \Lambda$ saturated $\}$. It is obvious that $1 / 2 \leq R_{c}<R^{(s)} \leq 1, R_{c}(n) \leq R_{L}(n)$ and that the subset of saturated Delone sets of $\mathbb{R}^{n}$ is included in $\bigcup_{R_{c} \leq R \leq R^{(s)}} X_{R}$. More precisely we have the following facts.

Lemma 3.2 i) $R^{(s)}=1$; ii) $R_{c}(n) \geq \frac{\sqrt{2}}{2} \sqrt{\frac{n}{n+1}}=\frac{\sqrt{2}}{2}(1+O(1 / n))$ for $n$ large.

Proof : i) Let us assume $R^{(s)}<1$ and that $R^{(s)}$ is the Delone constant of a saturated Delone set $\Lambda$. We will obtain a contradiction. Then there exists $z \in \mathbb{R}^{n}$ such that $\inf _{\lambda \in \Lambda}\|z-\lambda\|=R^{(s)}$. Up to a translation, we may assume $z=0$. Let $\epsilon>0$ be small enough such that $(1+\epsilon) R^{(s)}<1$. Let $\eta \in(3,4)$ such that the system of spheres $\mathcal{B}\left(\Lambda \cap B\left(0, \eta R^{(s)}\right)\right):=$ $\left\{B\left(c_{1}, 1 / 2\right), \ldots, B\left(c_{m}, 1 / 2\right)\right\} \quad$ (with $m \geq 1$ ) is such that $\left\|h c_{j}\right\|<\eta R^{(s)}$ for all $j=1,2, \ldots, m$ and all $h \in[1,1+\epsilon)$.

Now let $h \in(1,1+\epsilon)$ and let us create the new Delone set $\Lambda_{h}$ from $\Lambda$ as follows: first, $\Lambda_{h} \cap B\left(0, \eta R^{(s)}\right)$ is exactly equal to the set $\left\{h c_{1}, h c_{2}, \ldots, h c_{m}\right\}$ so that, "inside" the ball $B\left(0, \eta R^{(s)}\right), \mathcal{B}\left(\Lambda_{h} \cap B\left(0, \eta R^{(s)}\right)\right)=\left\{B\left(h c_{1}, 1 / 2\right), \ldots, B\left(h c_{m}, 1 / 2\right)\right\}$. For constructing $\mathcal{B}\left(\Lambda_{h} \cap\left(\mathbb{R}^{n} \backslash B\left(0, \eta R^{(s)}\right)\right)\right)$, we take any infinite packing $\mathcal{B}_{1}$ of balls of radius $1 / 2$ centred at points which lie in $\mathbb{R}^{n} \backslash B\left(0, \eta R^{(s)}\right)$ so that: i) $\mathcal{B}_{1} \cup \mathcal{B}\left(\Lambda_{h} \cap B\left(0, \eta R^{(s)}\right)\right)$ is a packing of balls of $\mathbb{R}^{n}$, and ii) $\mathcal{B}_{1}$ is saturated. We obtain the Delone set $\Lambda_{h} \in \mathcal{U D}$ defined by $\mathcal{B}\left(\Lambda_{h}\right):=$ $\mathcal{B}_{1} \cup \mathcal{B}\left(\Lambda_{h} \cap B\left(0, \eta R^{(s)}\right)\right)$.

We will take $\epsilon$ small enough such that all the Voronoi cells at the points $h c_{j}$, with $j=1,2, \ldots, m$ and $h \in[1,1+\epsilon)$, have a circumradius always strictly less than 1 (this is always possible because of the continuity of the maps defining the vertices of Voronoi cells as functions of the centres of balls). Since the restriction of the system of balls $\mathcal{B}\left(\Lambda_{h}\right)$ to the portion of space outside the cluster $\left\{B\left(h c_{1}, 1 / 2\right), \ldots, B\left(h c_{m}, 1 / 2\right)\right\} \cup B\left(0, \eta R^{(s)}\right)$, is saturated, all the Voronoi cells at the centres of the balls of $\mathcal{B}_{1}$ have a circumradius $\leq R^{(s)}<1$.

Then, on one hand, since the distance between 0 and $\Lambda_{h}$ is $h R^{(s)}>$ $R^{(s)}$, for $h>1$, the Delone set $\Lambda_{h}$ has a Delone constant strictly greater 
than $R^{(s)}$. Hence it is not saturated, by definition of $R^{(s)}$. On the other hand, since all the Voronoi cells of $\Lambda_{h}$, at the centres of balls located "outside" and "inside" $B\left(0, \eta R^{(s)}\right)$, have a circumradius strictly less than 1 , it is impossible to add a sphere at any of their vertices to saturate $\Lambda_{h}$, and therefore there is no place in $\mathbb{R}^{n}$ where to add a ball of radius $1 / 2$ to saturate $\Lambda_{h}$. Contradiction.

In the case where the supremum $R^{(s)}=\sup \{R(\Lambda) \mid \Lambda$ saturated $\}$ is not reached, let us still assume that $R^{(s)}<1$ and let us show the contradiction. Then, necessarily [Verger-Gaugry 2001], there exist a sequence of points $\left(z_{i}\right)_{i \geq 1}$ and a sequence of Delone sets $\left(\Lambda_{i}\right)_{i \geq 1}$ such that: $\left\|z_{i}\right\|$ tends to $+\infty$ when $i$ goes to infinity with the property that, for all $\epsilon>0$ there exists $i_{0}(\epsilon)$ such that $i \geq i_{0}(\epsilon)$ implies $R^{(s)}-\epsilon \leq \inf _{\lambda \in \Lambda_{i}}\left\|z_{i}-\lambda\right\| \leq$ $R^{(s)}$. Let $R_{i}$ be the Delone constant of $\Lambda_{i}$. We now take $\epsilon$ small enough in order to have $1 / R^{(s)}>1 /\left(1-\epsilon / R^{(s)}\right)$. It corresponds to values of $i$ large enough. Then, as above, we will consider a new Delone set $\Lambda_{h, i}$ created from $\Lambda_{i}$ by a local dilation of scalar factor $h$ about the point $z_{i}$. When $1 / R^{(s)}>h>1 /\left(1-\epsilon / R^{(s)}\right)$ then $h R_{i} \leq h R^{(s)}<1$ and $h R_{i} \geq h\left(R^{(s)}-\epsilon\right)>\left(R^{(s)}-\epsilon\right) /\left(1-\epsilon / R^{(s)}\right)=R^{(s)}$. As above, we obtain a Delone set $\Lambda_{h, i}$ which is such that its Delone constant is strictly greater than $R^{(s)}$ and strictly smaller than 1 , then not saturated and impossible to saturate. Contradiction.

ii) Let us show that, if $\Lambda$ is a Delone set of $\mathbb{R}^{n}$ of constant $R, n \geq 1$, then $\frac{\sqrt{2}}{2} \sqrt{\frac{n}{n+1}} \leq R$. This inequality comes from an inequality of Blichfeldt (Lemma 1 in [Rogers 1964] p 79; or [Blichfeldt 1929]) since the distance from the centre of a Voronoi cell to any point of its $(n-i)$-dimensional plane, in the Voronoi decomposition of space by $\Lambda$, is at least $\frac{1}{2} \sqrt{\frac{2 i}{i+1}}$ for all $1 \leq i \leq$ $n$. Taking $i=n$ in the above inequality gives the result. Note that in the constructions of Rogers, packings of equal ball of radius 1 , and not $1 / 2$, are considered; this justifies the factor $1 / 2$ in front of the expression.

We will call $\sqrt{2} / 2$ the Blichfeldt bound.

If $n=1, X_{R_{c}}=X_{1 / 2}$ is not empty since it contains $\mathbb{Z}$. If $n=2$, the set $X_{R_{c}}=X_{\frac{1}{\sqrt{3}}}$ is not empty since it contains the lattice generated by the points of coordinates $(1,0)$ and $(1 / 2, \sqrt{3} / 2)$ in the plane (extreme lattice) in an orthonormal basis [Kerschner 1939]. What happens for $n \geq 3$ ? The set $X_{\frac{\sqrt{2}}{2} \sqrt{\frac{n}{n+1}}}$ is certainly empty since the minimal Voronoi cell is not tiling periodically the ambiant space as soon as $n \geq 3$ [Rogers 1964] (McLaughlin's Theorem cited in [Hales 2000], Hales [HalesI 1997] [HalesII 1997] for $n=3$, [Oesterlé 1998/9], [Verger-Gaugry 2001]). 
$\left(\mathrm{Q}_{2}\right)$ For which values of $n$ and $R$ is $X_{R}$ not empty?

This fairly old question [Ryshkov 1975] is partially answered by Theorem 1.2 .

\section{PROOFS OF THEOREMS 1.1 AND 1.2}

Proof of Theorem 1.1: Let $R_{c} \leq R$ and $T>R$ be a real number. If $\Lambda$ is a Delone set of constant $R$ of $\mathbb{R}^{n}$, then $(B(0, R)+\Lambda) \cap B(0, T)$ covers the ball $B(0, T-R)$. Hence, the number of elements of $\Lambda \cap B(0, T)$ is at least $((T-R) / R)^{n}$. On the other hand, since all the balls of radius $1 / 2$ centred at the elements of $\Lambda \cap B(0, T)$ lie within $B(0, T+1 / 2)$, the proportion of space they occupy in $B(0, T+1 / 2)$ is at least

$$
\left(\frac{T-R}{R}\right)^{n} \frac{\operatorname{vol}(B(0,1 / 2))}{\operatorname{vol}(B(0, T+1 / 2))}=\left(\frac{T-R}{2 R(T+1 / 2)}\right)^{n} .
$$

When $T$ tends to infinity the above quantity tends to $(2 R)^{-n}$ which is a lower bound of the density $\delta(\mathcal{B}(\Lambda))$.

Proof of Theorem 1.2: Let $\sigma_{K L}(n)=2^{-0.599 n}$ be the upper bound of Kabatjanskii-Levenštein of the packing density $\delta$. By Theorem 1.1 we deduce that, with $R_{c} \leq R \leq 1$,

$$
\mu_{n}(R) \leq \delta \leq 2^{-0.599 n}
$$

Raising this equation to the power $1 / n$ gives readily $2 R \geq 2^{0.599}+o(1)$ that is $R \geq 2^{-0.401}+o(1)$.

\section{ASYMPTOTIC BEHAVIOUR OF HOLES IN SEQUENCES OF LATTICES AND PACKINGS}

The expression of the bound $\mu_{n}(R)$ will be used to compute a lower bound of the Delone constant of a Delone set, or a lower bound of the covering radius of a given lattice $L \in \mathcal{U D} \cap \mathcal{L}$, when its density and its minimal interpoint distance are known.

In the case of a lattice $L$, the minimal interpoint distance of $L$ is the square root of the norm $N(L)$ of the lattice [Martinet 1996]. We will consider the normalized lattice $\frac{1}{\sqrt{N(L)}} L$ instead of the lattice $L$ to apply the preceding considerations with packings of spheres of common radius $1 / 2$. The situation is similar for a Delone set which will be normalized by its minimal interpoint distance. We will denote by $\operatorname{dens}(L):=$ 
$\delta(\mathcal{B}(L / \sqrt{N(L)}))$ (Theorem 1.7 in [Rogers 1964]) the density of the system of spheres $L+B(0, \sqrt{N(L)} / 2)$ if $L$ is a lattice and by $\operatorname{dens}(\Lambda):=$ $\delta(\mathcal{B}(\Lambda / n(\Lambda))$ ) (Theorem 1.7 in [Rogers 1964]) the density of the system of spheres $\Lambda+B(0, n(\Lambda) / 2)$ if $\Lambda$ is a Delone set of minimal interpoint distance $n(\Lambda)$.

Let us observe that, for all Delone set $\Lambda$ and all nonnegative scalar factor $\lambda$ such that $\Lambda \in \mathcal{U D}$ and $\lambda \Lambda \in \mathcal{U D}$, the equality $R(\lambda \Lambda)=\lambda R(\Lambda)$ holds. Then, from Theorem 1.1, we readily obtain the following inequalities: i) $\frac{n(\Lambda)}{2} \operatorname{dens}(\Lambda)^{-1 / n} \leq R(\Lambda)$, for all Delone set $\Lambda \in \mathcal{U} \mathcal{D}$ of minimal interpoint distance $n(\Lambda)$, and ii) $\frac{\sqrt{N(L)}}{2} \operatorname{dens}(L)^{-1 / n} \leq R(L)$, for all lattice $L \in \mathcal{U D} \cap \mathcal{L}$ of norm $N(L)$. In the sequel the following notations will be used: $t_{L}:=\sqrt{N(L)} \tilde{t}_{L}$ with $\tilde{t_{L}}:=\frac{1}{2} \operatorname{dens}(L)^{-1 / n}$; and $t_{\Lambda}:=n(\Lambda) \tilde{t_{\Lambda}}$ with $\tilde{t_{\Lambda}}:=\frac{1}{2} \operatorname{dens}(\Lambda)^{-1 / n}$ for $L$ and $\Lambda$ as above.

Let us now apply these inequalities to some known sequences of lattices and packings, as given by [Conway - Sloane 1988] Chap. 5 and 8, and [Martinet 1996] Chap. V, to obtain an estimation of the size of the deep holes.

\subsection{LEECH LATTICE}

For the Leech lattice $\Lambda_{24}$ in $\mathbb{R}^{24}$ the density $\delta\left(\Lambda_{24}\right)=\pi^{12} / 479001600=$ $0.001930 \ldots$ and the covering radius $R\left(\Lambda_{24} / \sqrt{N\left(\Lambda_{24}\right)}\right)=\sqrt{2} / 2$ are both known [Conway - Sloane 1988] [ElkiesI 2000]. We obtain $t_{\Lambda_{24}}=0.6487 \ldots$ This numerical value is less than $10 \%$ far from the true value $0.707 \ldots$. This estimation of the size of the deep hole in $\Lambda_{24}$ is fairly realistic.

\subsection{BARNES-WALL LATTICES}

The density of the Barnes-Wall lattice $B W_{n}$ ([Leech 1964], [Conway - Sloane 1988] p 234 or p 151), in $\mathbb{R}^{n}, n=2^{m}, m \geq 2$, is equal to $2^{-5 n / 4} n^{n / 4} \pi^{n / 2} / \Gamma(1+$ $n / 2$ ). The norm $N\left(B W_{n}\right)$ is equal to $n$ ([Leech 1964] p 678).

Proposition 5.1 Let $n=2^{m}$ with $m \geq 2$. The covering radius $R\left(B W_{n}\right) \geq$ $t_{B W_{n}}$ of the Barnes-Wall lattice $B W_{n}$ is such that the size of its (deepest) hole tends to infinity as (and better than)

$$
t_{B W_{n}}:=\frac{2^{-1 / 4}}{\sqrt{\pi e}} n^{3 / 4}(1+o(1))
$$

when $n$ goes to infinity. 
Proof : Raising to the power $1 / n$ the equation

$$
2^{-5 n / 4} n^{n / 4} \pi^{n / 2} / \Gamma(1+n / 2)=\delta\left(\mathcal{B}\left(B W_{n} / \sqrt{n}\right)\right)=\mu_{n}(t)
$$

and allowing $n$ to tend to infinity leads easily to the claimed asymptotic expression of $t_{B W_{n} / \sqrt{n}}$ as a function of $n$. The multiplication of $t_{B W_{n} / \sqrt{n}}=\tilde{t}_{B W_{n}}$ by the minimal interpoint distance $\sqrt{n}$ gives the claimed lower bound $t_{B W_{n}}$ of the covering radius $R\left(B W_{n}\right)$ of $B W_{n}$.

\subsection{BCH PACKINGS}

In this subsection, the reference will be [Conway - Sloane 1988] p 155. Let $n=2^{m}, m \geq 4$. The packings of equal spheres considered below are obtained using extended $\mathrm{BCH}$ codes in construction $\mathrm{C}$ of length $n$. They are not lattices. There are two packings ( $\mathrm{a}$ and $\mathrm{b}$ ) which use two different codes of the Hamming distances. Let us denote the second one by $P_{n b}$. Its density $\operatorname{dens}\left(P_{n b}\right)$ satisfies

$$
\log _{2} \operatorname{dens}\left(P_{n b}\right) \simeq-\frac{1}{2} n \log _{2} \log _{2} n \quad \text { as } n \rightarrow+\infty
$$

and its minimal interpoint distance is ([Conway - Sloane 1988] p 150) $n\left(P_{n b}\right)=$ $\sqrt{\gamma} 2^{a}$ with $\gamma=2$ and $a=[(m-1) / 2]$. We deduce

Proposition 5.2 Let $n=2^{m}$ with $m \geq 4$. The Delone constant $R\left(P_{n b}\right) \geq$ $t_{P_{n b}}$ of the BCH packing $P_{n b}$ tends to infinity as (and better than)

$$
t_{P_{n b}}=2^{-\frac{1}{2}+\left[\left(-1+\log _{2} n\right) / 2\right]} \sqrt{\log _{2} n}(1+o(1)) \simeq \frac{1}{\sqrt{2}} \log _{2} n(1+o(1))
$$

when $n$ goes to infinity.

The proof can be made with the same arguments as in the proof of Proposition 5.1.

\subsection{CRAIG LATTICES}

These lattices are known to be among the densest ones (Martinet [Martinet 1996] p 163-171, Conway and Sloane [Conway - Sloane 1988] p 222-224). The density $\operatorname{dens}\left(\mathbb{A}_{n}^{(r)}\right)$ of the Craig lattice $\mathbb{A}_{n}^{(r)}, n \geq 1, r \geq 1$, in $\mathbb{R}^{n}$ is at least

$$
\frac{(r / 2)^{n / 2}}{(n+1)^{r-1 / 2}} \frac{\pi^{n / 2}}{\Gamma(1+n / 2)}
$$


with equality if the norm of the lattice is $2 r$. The norm of Craig lattices is not known in general and lower bounds of $N\left(\mathbb{A}_{n}^{(r)}\right)$ were obtained by Craig [Martinet 1996] [Bachoc-Batut 1992] [Craig 1978]. The determination of $N\left(\mathbb{A}_{n}^{(r)}\right)$ is equivalent to the so-called Tarry-Escott problem in combinatorics and does not seem to be solved yet. However, for some values of $n$ and $r$ this norm is known.

Theorem 5.3 Let $n \geq 2$. (i) (Craig) If $n+1$ is a prime number $p$ and $r<n / 2$, then $N\left(\mathbb{A}_{n}^{(r)}\right) \geq 2 r$. (ii) (Bachoc and Batut) If $n+1$ is a prime number $p$ with $r$ a strict divisor of $n=p-1$, then $N\left(\mathbb{A}_{n}^{(r)}\right)=2 r$.

Bachoc and Batut [Bachoc-Batut 1992] made an exhaustive investigation of Craig lattices for the prime numbers $p \leq 23$. The equality $N\left(\mathbb{A}_{p-1}^{(r)}\right)=$ $2 r$ holds for $r=1, r=2, r=3$ and also for $r=(p+1) / 4$ with $p \equiv 3 \bmod 4$. This last case was proved by Elkies (cited in Gross [Gross 1990]), from the general theory of Mordell-Weil lattices developped by Elkies and Shioda concerning the groups of rational points of elliptic curves over function fields [Shioda 1992]. The equality $N\left(\mathbb{A}_{p-1}^{(r)}\right)=2 r$ was also proved to be true for $p \leq 37$ and $r \in\left[1, \frac{p+1}{4}\right]$ ([Martinet 1996] p 169), but wrong for higher values of $p$.

Using the assertion (ii) in Theorem 5.3 we obtain the following Proposition.

Proposition 5.4 let $n \geq 2$ such that $n+1$ is a prime number and $r$ a strict divisor of $n$. Then, the covering radius $R\left(\mathbb{A}_{n}^{(r)}\right) \geq t_{\mathbb{A}_{n}^{(r)}}$ of the Craig lattice $\mathbb{A}_{n}^{(r)}$ is such that the size of its (deepest) hole tends to infinity as (and better than)

$$
t_{\mathbb{A}_{n}^{(r)}}:=\frac{1}{\sqrt{2 \pi e}} \sqrt{n}(1+o(1))
$$

when $n$ goes to infinity.

Let us remark that $t_{\mathbb{A}_{n}^{(r)}}$ is independent of $r$ when $n$ is large enough. As shown by Propositions 5.1 and 5.4 the deep holes of the Barnes-Wall and Craig lattices $B W_{n}$ and $\mathbb{A}_{n}^{(r)}$ have a size which goes to infinity with $n$ ( $r$ fixed). In order to allow comparison between them and with Butler's Theorem (Theorem 3.1), we have to consider the normalized lattices

$$
\frac{1}{\sqrt{n}} B W_{n} \quad \text { and } \quad \frac{1}{\sqrt{2 r}} \mathbb{A}_{n}^{(r)}
$$


assuming that $n$ is such that $n+1$ is a prime number. In the first case, the covering radius tends to infinity with $n$ leaving no hope to obtain very dense packings of spheres from the lattices $B W_{n}$ when $n$ is large enough. In the second case, since

$$
t_{\mathbb{A}_{n}^{(r)} / \sqrt{2 r}}=\frac{1}{2 \sqrt{\pi e}} \sqrt{\frac{n}{r}}
$$

we see that $t_{\mathbb{A}_{n}^{(r)} / \sqrt{2 r}}>1$ if $r<\frac{1}{4 \pi e} n$. Let us recall, from Theorem 3.1 , that the existence of very dense lattices (of minimal interpoint distance one) of covering radius as close as 1 is expected. Therefore we can expect to find very dense Craig lattices satisfying this condition when $r=r(n)$ is a suitable function of $n$ and large enough, namely: $r(n)>\frac{1}{4 \pi e} n$ for which the lower bound $t_{\mathbb{A}_{n}^{(r)} / \sqrt{2 r}}$ of $R\left(\mathbb{A}_{n}^{(r)} / \sqrt{2 r}\right)$ is then less than unity. On the other hand, the density $\operatorname{dens}\left(\mathbb{A}_{n}^{(r)}\right)$ reaches its maximum when $r$ is the integer the closest to $\frac{n}{2 \ln (n+1)}$ (obtained by cancelling the derivative of $\operatorname{dens}\left(\mathbb{A}_{n}^{(r)}\right)$ with respect to $r$, with $n$ fixed, assuming that the norm of the lattice $\mathbb{A}_{n}^{(r)}$ is exactly $\left.2 r\right)$.

Since $\frac{n}{2 \ln (n+1)} \leq \frac{1}{4 \pi e} n$ as soon as $n$ is large enough (for $n \geq e^{2 \pi e}-1$ ), a good compromise for the value of $r$, assuming that the norm of the lattice $\mathbb{A}_{n}^{(r)}$ is exactly $2 r$, would be $r:=$ the smallest integer $>\frac{1}{4 \pi e} n$.

$\left(\mathrm{Q}_{3}\right)$ Does there exist normalized Craig lattices $\mathbb{A}_{n}^{(r)} / \sqrt{N\left(\mathbb{A}_{n}^{(r)}\right)}$ (for general $n$ and $r$ ) which exhibit a Delone constant (covering radius) smaller than 1 ?

\subsection{MORDELL-WEIL LATTICES}

We will refer here to the class of Mordell-Weil lattices given by the following Theorem of Shioda ([Shioda 1991] Theorem 1.1).

Theorem $5.5($ Shioda $)$ Let $p$ be a prime number such that $p+1 \equiv 0(\bmod 6)$ and $k$ any field containing $\mathbb{F}_{p^{2}}$. The Mordell-Weil lattice $E(K)$ of the elliptic curve $E$

$$
y^{2}=x^{3}+1+u^{p+1}
$$

defined over the rational function field $K$, where $K=k(u)$, is a positive- 
definite even integral lattice with the following invariants:

$$
\begin{aligned}
\text { rank } & =2 p-2 \\
\text { det } & =p^{\frac{p-5}{3}} \\
N(E(K)) & =\frac{p+1}{3} \\
\text { centre density } \Delta & =\frac{\left(\frac{p+1}{12}\right)^{p-1}}{p^{(p-5) / 6}} \\
\text { kissing number } & \geq 6 p(p-1)
\end{aligned}
$$

Recall that the centre density $\Delta$ is the quotient of the density of the lattice by the volume $\pi^{n / 2} / \Gamma(1+n / 2)$ of the unit ball of $\mathbb{R}^{n}$. Such a lattice in $\mathbb{R}^{2 p-2}$, denoted by $M W_{n}$ with $n=2 p-2$, has a minimal interpoint distance equal to $\sqrt{(p+1) / 3}$ and a density equal to $\operatorname{dens}\left(M W_{n}\right)=\Delta \frac{\pi^{p-1}}{\Gamma(p)}$. We deduce that

$$
\begin{aligned}
& \tilde{t}_{M W_{n}} \simeq \frac{1}{2} \frac{\sqrt{\pi}}{(\Gamma(p))^{1 /(2 p-2)}} \frac{\left(\frac{p+1}{12}\right)^{1 / 2}}{p^{(p-5) /(12(p-1))}} \\
& \simeq \frac{\sqrt{\pi e}}{4 \sqrt{3}} p^{-1 / 12} \simeq 2^{-2+1 / 12} \frac{\sqrt{\pi e}}{\sqrt{3}} n^{-1 / 12} .
\end{aligned}
$$

This value goes to zero while

$$
t_{M W_{n}} \simeq 2^{1 / 12} \frac{\sqrt{\pi e}}{12 \sqrt{2}} n^{5 / 12}
$$

goes to infinity when $p$ (or $n$ ) tends to infinity. This result indicates that the deep holes of the normalized Mordell-Weil lattice $M W_{n} / \sqrt{N\left(M W_{n}\right)}$ are in fact very shallow, and may be probably bounded above independently of $n$. This leads to the following question.

$\left(\mathrm{Q}_{4}\right)$ Does there exist normalized Mordell-Weil lattices $M W_{n} / \sqrt{N\left(M W_{n}\right)}$ which exhibit a Delone constant (covering radius) smaller than 1 ?

\section{COMMENTS AND CONJECTURE}

The lower bound $\mu_{n}(R)$ of $\delta$ is particularly interesting for Delone sets of Delone constant $R$ of $\mathbb{R}^{n}$ which are saturated, that is for $R \leq R^{(s)}$. Since $R^{(s)}=1$ by Lemma 3.2, we readily obtain a lower bound for $\delta$ which is $2^{-n} \quad$ [ElkiesI 2000] [ElkiesII 2000]. More generally, the lower bound $\mu_{n}(R)$ exhibits a dependence with $n$ which is in

$$
(2 R)^{-n}=2^{-n\left(1+\log _{2} R\right)} .
$$


Now taking $R=R^{(s)}=1$ gives a $2^{-n}$ dependence typical of the Minkowski-Hlawka type lower bounds of $\delta_{L}$ while taking $R=\sqrt{2} / 2$ (the Blichfeldt bound, Lemma 3.2) provides a $2^{-n / 2}$ dependence typical of the Rogers bound $\sigma_{n}$. Inbetween all values of $R$ are formally possible but the range is limited (Theorem 1.2).

Here the viewpoint is not that of explicit constructions. Working with packings of spheres arising from Delone sets for which we only control the constant $R$ would seem a priori to give more freedom to the constructions. Very dense packings are likely to occur with 'almost-touching' spheres everywhere, that is from Delone sets of Delone constants $R$, as small as possible, close to $R_{c}(n)$. The corresponding kissing numbers deduced from all the local clusters of spheres would lie between the Coxeter-Böröczky/ Kabatjanskii-Levenštein upper bounds [Böröczky 1978] [Kabatjanskii - Levenštein 1978] and the lower bound of Wyner [Wyner 1965] and probably closer to their upper bounds. Local arrangements of spheres in a densest sphere packing can be extremely diversified ([Hales 2000] [HalesI 1997] [HalesII 1997] on Hales-Ferguson Theorem, for $n=3)$.

In this sense, Theorem 1.1 gives a partial answer to old expectations when $R$ lies between $R_{c}(n)$ and 1. Indeed, recall [Gruber - Lekkerkerker 1987] p 391 : "the best known upper and lower bounds for $\delta$ differ by a factor which is approximately $2^{n / 2}$. This means that the problem of closest packing of spheres is still far from its solution (except for low values of $n$ )".; and [Rogers 1964] p 9 : we were still up till now in the situation where "There remains a wide gap between the results of the Minkowski-Hlawka type, ..., and the results of Blichfeldt type, ...". 


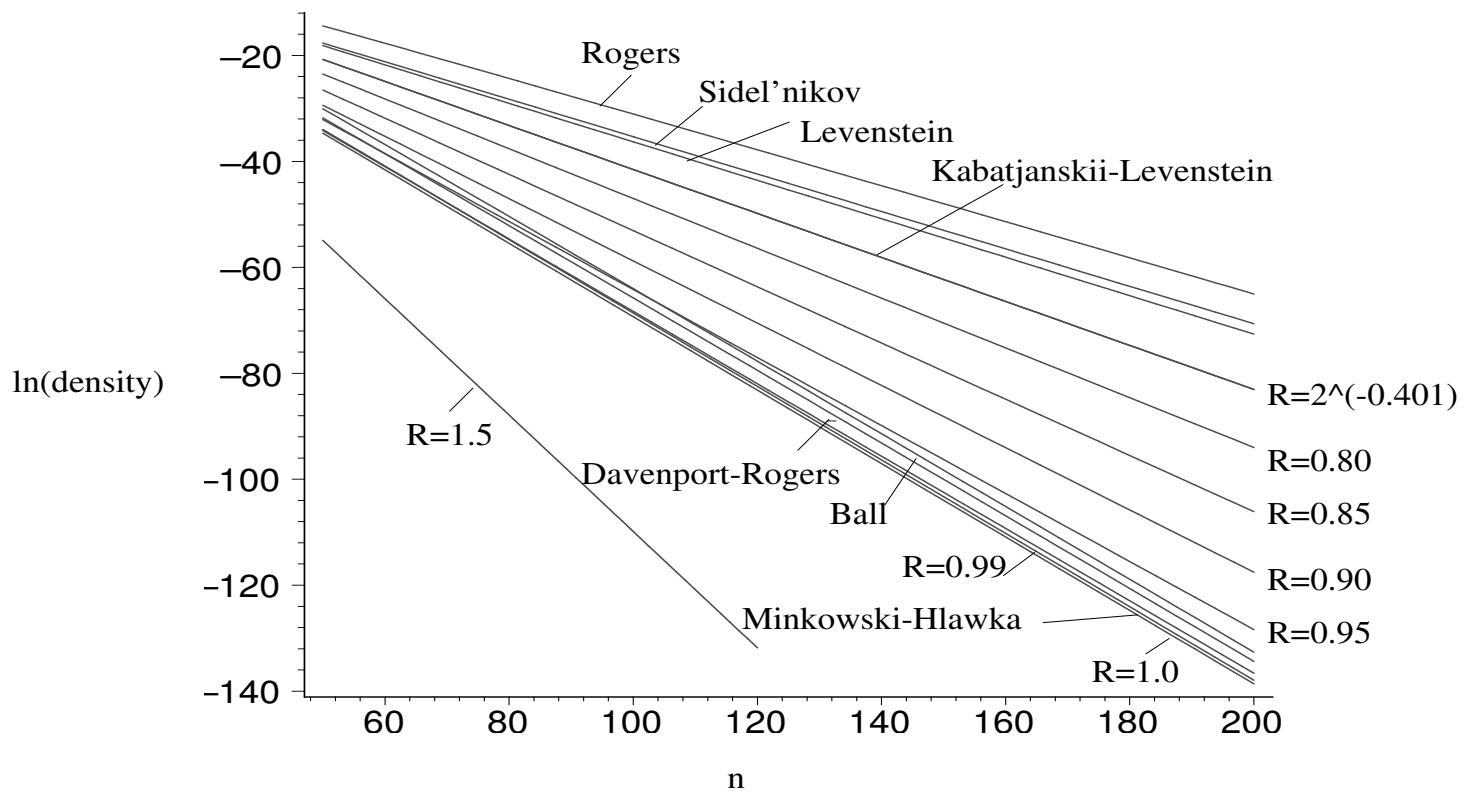

Figure 1: Upper bounds of the packing density $\delta$ and lower bounds of the lattice-packing density $\delta_{L}$. The $R$-dependent lower bounds $\mu_{n}(R)$ are plotted for $R=2^{-0.401}, 0.8,0.85,0.90,0.95,0.99,1.5$ as a function of the dimension $n$.

In Figure 1 are plotted the $R$-dependent bound $\mu_{n}(R)$ for several values of $R$, the upper bounds of Rogers, Sidel'nikov, Levenštein, KabatjanskiiLevenštein, the lower bounds of Davenport-Rogers, Ball and of MinkowskiHlawka, as a function of the dimension $n$. All values between these two types of bounds can be reached by $\mu_{n}(R)$ when $R$ is suitably chosen below 1.

The curve $n \rightarrow \mu_{n}(R)$ for $R=1$ is slightly below the MinkowskiHlawka bound. When $R$ is greater than 1 , the curves $n \rightarrow \mu_{n}(R)$ are entirely below the Minkowski-Hlawka bound. On the contrary, when $R<$ 1 is close to unity, the curve $\mu_{n}(R)$ lies below the Minkowski-Hlawka bound up till a certain value of $n$ and then dominates it, as expected asymptotically. When $2^{-0.401}<R<1$ lies far enough from 1 the entire curve $n \rightarrow \mu_{n}(R)$ lies strictly between the two types of bounds (Kabatjanskii-Levenštein and Minkowki-Hlawka).

Theorem 1.2 does not say anything about the frequency and the density of such middle-sized Voronoi cells of circumradius $R$ approximately equal to $2^{-0.401}$ in a general saturated Delone set of $\mathbb{R}^{n}$ of constant $R$ when $n$ is sufficiently large, in particular in the densest ones. 


\begin{tabular}{|ccc|} 
Type & Name & $\log _{2} \Delta$ \\
\hline constructions & Barnes-Wall $B W_{65536}$ & 180224 \\
& $B_{65536}$ & 290998 \\
& $\eta\left(\Lambda_{32}\right)$ & 295120 \\
& Craig $\mathbb{A}_{65536}^{(2954)}$ & 297740 \\
\hline (existence) lower bounds & Minkowski-Hlawka & 324603 \\
of $\delta_{L}$ & Davenport-Rogers & 324616 \\
& Ball & 324620 \\
\hline$\mu_{65536}(R)$ & $R=1.5$ & 286266 \\
& $R=1.0$ & 324602 \\
lower bounds & $R=0.99$ & 325553 \\
from Theorem 1.1 & $R=0.95$ & 329452 \\
& $R=0.90$ & 334564 \\
& $R=0.85$ & 339968 \\
& $R=0.80$ & 345700 \\
& $R=2^{-0.401}$ & 350882 \\
\hline upper bounds & Kabatjanskii-Levenšstein & 350882 \\
& Levenštein & 355818 \\
of $\delta$ & Sidel'nikov & 356742 \\
& Rogers & 357385
\end{tabular}

Table 3 : Table 1.4 of [Conway - Sloane 1988] Chap. 1 to which we have added the lower bounds $\mu_{65536}(R)$ for different values of $R$ (the values of the centre density $\log _{2} \Delta$ are recomputed from the original references).

To allow comparison with known results in literature and to follow Conway and Sloane [Conway - Sloane 1988] we have taken $n$ fairly large, namely $n=65536$. To appreciate the pertinency of the formula given by Theorem 1.1 we have reproduced in Table 3 the Table 1.4 of [Conway - Sloane 1988] Chap. 1 and added therein the values of the centre density $\Delta$ deduced from $\mu_{65536}(R)$ for $R=2^{-0.401}, 0.8,0.85,0.90,0.95,0.99,1.0,1.5$. The value of (the logarithm in base 2 of) the centre density $\Delta$ computed from $\mu_{65536}(R)$ now sticks to the Kabatjanskii-Levenštein's bound when $R$ is at its asymptotic maximum $R=2^{-0.401}$. Is this value reached by the Delone constant of a Delone set?

When $n$ is large enough, the sensitivity of $\mu_{n}(R)$ to the Delone constant $R$ can be perceived by the following comparison (see Table 3 ): the centre density 324602 relative to the bound $\mu_{65536}(1)$ is slightly below the lower bound 324603 of Minkowski-Hlawka, as expected, whereas the centre density 325553 relative to $\mu_{65536}(0.99)$ is slightly above the best lower bound 324620 of Ball. This gives credit to the Conjecture (see 
question $\mathrm{Q}_{1}$ for a precise formulation) that lattices do not exhibit a covering radius less than 1 when $n$ is sufficiently large.

\section{ACKNOWLEDGMENTS}

The authors would like to thank Roland Bacher for very helpful comments and suggestions.

\section{References}

[Bachoc-Batut 1992] C. BACHOC AND C. BATUt, Etude algorithmique de réseaux construits avec la forme trace, Experiment. Math., 1, (1992), 183-190.

[Ball 1992] K. BALL, A lower bound for the optimal density of lattice packings, Int. Math. Res. Notices, 10, (1992), 217-221.

[Blichfeldt 1929] H.F. BLICHFELDT, The minimum value of quadratic forms, and the closest packing of spheres, Math. Ann. 101, (1929), 605-608.

[Böröczky 1978] K. BöRÖCzKY, Packings of spheres in space of constant curvature, Acta Math. Acad. Sci. Hung. 32, (1978), 243-261.

[Böröczky 1986] K. BöRÖCzKY, Closest packing and loosest covering of the space with balls, Studia Sci. Math. Hungar., 21, (1986), 79-89.

[Butler 1972] G.J. ButleR, Simultaneous packing and covering in Euclidean space, Proc. London Math. Soc., 25, (1972), 721-735.

[Cassels 1959] J.W.S. CAssels, An introduction to the Geometry of Numbers, Springer Verlag, (1959).

[Coxeter 1963] H.S.M. COXETER, An upper bound for the number of equal nonoverlapping spheres that can touch another of the same size, Proc. Symp. Pure Math., "Convexity", Vol. VII, A.M.S. Providence, (1963), 53-71.

[Conway - Sloane 1988] J.H. Conway And N.J.A. SloAne, Sphere packings, lattices and groups, Springer-Verlag, (1988).

[Craig 1978] M. Craig, Extreme forms and cyclotomy, Mathematika, 25, (1978), 44-56.

[Davenport - Rogers 1947] H. Davenport And C.A. Rogers, Hlawka's Theorem in the geometry of numbers, Duke Math. J., 14, (1947), 367-375.

[ElkiesI 2000] N.D. ElKIES, Lattices, linear codes and invariants. I., Notices Amer. Math. Soc. 47, n10, (2000), 1238-1245.

[ElkiesII 2000] N.D. ElkiES, Lattices, linear codes and invariants. II., Notices Amer. Math. Soc. 47, n11, (2000), 1382-1391. 
[Fejes-Toth 1979] G. FEJEs-Tóth, Multiple packing and covering of spheres, Acta Math. Acad. Sci. Hungar., 34, (1979), 165-176.

[Gross 1990] B. Gross, Group representation and lattices, J. Amer. Math. Soc., 3, (1990), 929-960.

[Gruber - Lekkerkerker 1987] P.M. Gruber AND C.G. LerkKerkerker, Geometry of Numbers, North-Holland, (1987).

[Hales 2000] T.C. HALES, Cannonballs and Honeycombs, Notices of the AMS, 47, n 4, (2000), 440-449.

[HalesI 1997] T.C. Hales, Sphere packings I, Disc. Comp. Geom. 17, (1997), 1-51.

[HalesII 1997] T.C. Hales, Sphere packings II, Disc. Comp. Geom. 18, (1997), 135-149.

[Handbook 1997] Handbook of Discrete and Computational Geometry, Ed. by J.E. Goodman and J.O'Rourke, CRC Press, Boca Raton, (1997), chap 2 and p 919.

[Henk 1995] M. HENK, Finite and infinite packings, Habilitationsschrift, Universität Siegen, (1995).

[Hoffstein 2001] J. Hoffstein, J. Pipher And J.H. Silvermann, NSS: an NTRU latticebased signature scheme, Advances in Cryptology - Eurocrypt 2001, Lecture Notes in Comput. Sci. 2045, (2001), 211-228.

[Horvath 1982] J. HoRvÁth, On close lattice packing of unit spheres in the space $E^{n}$, in 'Geometry of positive quadratic forms', Proc. Steklov Math. Inst. 152, (1982), 237-254.

[Kerschner 1939] R. KenschneR, The number of circles covering a set, Amer. J. Math. 61, (1939), 665-671.

[Kabatjanskii - Levenštein 1978] G.A. KabatjanskiĬ And V.I. LevenšTein, Bounds for packings on a sphere and in space, Problems of Information Transmission, 14, (1978), $1-17$.

[Leech 1964] J. LeEch, Some sphere packings in higher space, Can. J. Math., 16, (1964), 657-682.

[Levenštein 1979] V.I. LEVENS̆TEIN, On bounds for packings in n-dimensional Euclidean space, Soviet Math. Dokl., 20, (1979), 417-421.

[Martinet 1996] J. MARTINET, Les réseaux parfaits des espaces euclidiens, Masson, (1996).

[Oesterlé 1989/90] J. Oesterlé, Empilements de sphères, Séminaire Bourbaki, n 727, (1989-90), 375-397; SMF Astérisque 189-190, (1990).

[Oesterlé 1998/9] J. Oesterlé, Densité maximale des empilements de sphères en dimension 3 [d'après Thomas C. Hales et Samuel P. Ferguson], Séminaire Bourbaki, n 863, (1998-99), 405-413. 
[Rogers 1964] C. A. Rogers, Packing and covering, Cambridge University Press, (1964).

[Rogers 1963] C. A. Rogers, Covering a sphere with spheres, Mathematika, 10, (1963), $157-164$.

[Rogers 1959] C. A. Rogers, Lattice coverings of space, Mathematika, 6, (1959), 33-39.

[Rogers 1958] C. A. Rogers, The packing of equal spheres, Proc. London Math. Soc., (3), 8, (1958), 609-620.

[Rogers 1950] C. A. Rogers, A note on coverings and packings, J. London Math. Soc., 25, (1950), 327-331.

[Rogers 1957] C.A. Rogers, A note on coverings, Mathematika, 4, (1957), 1-6.

[Ryshkov 1975] S.S. RYshkov, Density of an ( $r, R)$-system, Math. Notes, 16, (1975), 855-858.

[Shioda 1992] T. SHIODA Some remarks on elliptic curves over functions fields, Astérisque, 209, (1992), 99-114.

[Shioda 1991] T. ShIOda Mordell-Weil lattices and sphere packings, Amer. J. Math., 113, (1991), 931-948.

[Sidel'nikov 1973] V.M. SIDEL'NIKOv, On the densest packing of balls on the surface of an n-dimensional Euclidean sphere and the number of binary code vectors with a given code distance, Soviet Math. Dokl., 14, (1973), 1851-1855.

[Verger-Gaugry 1997] J.-L. Verger-Gaugry, On a generalization of the Hermite constant, Periodica Mathematica Hungarica, 34, (1997), 153-164.

[Verger-Gaugry 2004] J.-L. VERGER-GAUGRY, Covering a ball with smaller equal balls in $\mathbb{R}^{n}$, Discr. Comp. Geom., 32 (4), (2004), to appear.

[Verger-Gaugry 2001] J.-L. Verger-Gaugry, Remarks on the Hales-Ferguson Theorem, Séminaire de Théorie Spectrale et Géométrie, Grenoble, 19, (2001), 165-177.

[Wyner 1965] A.D. Wyner, Capabilities of bounded discrepancy decoding, Bell System Tech. J., 44, (1965), 1061-1122.

[Zong 1999] C. ZoNG Sphere packings, Springer-Verlag, (1999).

G. Muraz, Institut Fourier, Université Grenoble I, UMR 5582 CNRS, BP 74, Domaine Universitaire, 38402 St Martin d'Hères Cedex, France (Gilbert.Muraz@ujf-grenoble.fr)

J.-L. Verger-Gaugry, Institut Fourier, Université Grenoble I, UMR 5582 CNRS, BP 74, Domaine Universitaire, 38402 St Martin d'Hères Cedex, France (jlverger@ujf-grenoble.fr) 